Annals of Pure and Applied Mathematics

Vol. 18, No. 1, 2018, 15-25

ISSN: 2279-087X (P), 2279-0888(online)

Published on 16 July 2018

Annals of

www.researchmathsci.org

DOI: http://dx.doi.org/10.22457/apam.v18n1a3

Pure and Applied

Mathematics

\title{
Case Study of Non Strong Arc in Cartesian Product of Fuzzy Graphs
}

\author{
C.Y. Ponnappan ${ }^{1}$ and V.Senthilkumar ${ }^{2}$ \\ ${ }^{1}$ Department of Mathematics, Government Arts College \\ Melur, Tamilndu -625106, email: Pons-mdu1969@ yahoo.com \\ ${ }^{2}$ Department of Mathematics, TKS College of Arts and Science, Theni \\ Tamilnadu-625534, email: 1984senthilvkumar@gmail.com \\ ${ }^{2}$ Corresponding author.
}

Received 26 May 2018; accepted 15 July 2018

Abstract. In this paper, we discussed the Cartesian product of the fuzzy graphs like fuzzy path and fuzzy cycle. We done case study of non strong arc in Cartesian product of fuzzy graph and deduced the formula for finding number of non strong arc in Cartesian product of the fuzzy graph.

Keywords: Fuzzy graph, Cartesian product, Strong arc, Non strong arc, Strong arc domination.

\section{AMS Mathematics Subject Classification (2010): $05 \mathrm{C} 72$}

\section{Introduction}

In1965, Zadeh introduced the concept of fuzzy subset of a set as a way for representing uncertainty [15]. Zadeh's ideas stirred the interest of researchers worldwide. Moderson and Nair discussed fuzzy graph theory fuzzy hypergraph [4]. Fuzzy graph is the generalization of the ordinary graph. The formal mathematical definition of domination was given by Ore in 1962 [10]. In 1975, Rosenfeld introduced the notion of fuzzy graph and several analogs of theoretic concepts such as path, cycle and connectedness [11]. Somasundaram and Somasundaram discussed the domination in fuzzy graph using effective arc[12]. Nagoorgani and Chandrasekarn discussed the strong arc in fuzzy graph $[8,9]$. Bhutani and Rosenfeld have introduced the concept of strong arcs in fuzzy graph $[1,2]$. Several works on fuzzy graph are also done by Pal and Rashmanlou [5,6], Methew and Sunitha [7], Samanta and Pal [14-20]. Before discuss case study of non strong arc in Cartesian product of fuzzy graph, we are placed few preliminary

\section{Preliminaries $[8,13]$}

Definition 2.1. Fuzzy graph $\mathrm{G}(\sigma, \mu)$ is pair of function, $V \rightarrow[0,1]$ and $\mu: V \times V \rightarrow[0,1]$ where for all $u, v$ in $V$, we have $\mu(u, v) \leq \sigma(u) \wedge \sigma(v)$.

Definition 2.2. The fuzzy graph $H(\tau, \rho)$ is called a fuzzy subgraph of $\mathrm{G}(\sigma, \mu)$ if $\tau(u) \leq$ $\sigma(u)$ for all $u$ in $V$ and $\rho(u, v) \leq \mu(u, v)$ for all $u, v$ in $V$. 


\section{C.Y. Ponnappan and V.Senthilkumar}

Definition 2.3. A fuzzy subgraph $H(\tau, \rho)$ is said to be a spanning sub graph of $\mathrm{G}(\sigma, \mu)$ if $\tau(u)=\sigma(u)$ for all $\mathrm{u}$ in $\mathrm{V}$. In this case the two graphs have the same fuzzy node set, they differ only in the arc weights.

Definition 2.4. Let $\mathrm{G}(\sigma, \mu)$ be a fuzzy graph and $\tau$ be fuzzy subset of $\sigma$, that is, $\tau(u) \leq$ $\sigma(u)$ for all $u$ in $V$. Then the fuzzy subgrpah of $\mathrm{G}(\sigma, \mu)$ induced by $\tau$ is the maximal fuzzy subgraph of $\mathrm{G}(\sigma, \mu)$ that has fuzzy node set $\tau$. Evidently, this is just the fuzzy graph $H(\tau, \rho)$ where $\rho(u, v)=\tau(u) \wedge \tau(v) \wedge \mu(u, v)$ for all $\mathrm{u}, \mathrm{v}$ in $\mathrm{V}$.

Definition 2.5. The underlying crisp graph of a fuzzy graph $\mathrm{G}(\sigma, \mu)$ is denoted by $G^{*}=\left(\sigma^{*}, \mu^{*}\right)$, where

$$
\sigma^{*}=\{u \in V \mid \sigma(u)>0\} \text { and } \mu^{*}=\{(u, v) \in V \times V \mid \mu(u, v)>0\} .
$$

Definition 2.6. A fuzzy graph $\mathrm{G}(\sigma, \mu)$ is a strong fuzzy graph if $\mu(u, v)=\sigma(u) \wedge$ $\sigma(v)$ for all $u, v \in \mu^{*}$ and is a complete fuzzy graph if

$$
\mu(u, v)=\sigma(u) \wedge \sigma(v) \text { for all } u, v \text { in } \sigma^{*} .
$$

Two nodes $\mathrm{u}$ and $\mathrm{v}$ are said to be neighbors if $\mu(u, v)>0$.

Definition 2.7. A fuzzy graph $\mathrm{G}=(\sigma, \mu)$ is said to be Bipartite if the node set $\mathrm{V}$ can be Partitioned into two non empty sets $V_{1}$ and $V_{2}$ such that $\mu(v 1, v 2)=0$ if $v_{1}, v_{2} \epsilon V_{1}$ or $\mathrm{v}_{1}, \mathrm{v} 2 \in \mathrm{V}_{2}$. Further if $\mu\left(\mathrm{v}_{1}, \mathrm{v}_{2}\right)>0$ for all $\mathrm{v}_{1} \in \mathrm{V}_{1}$ and $\mathrm{v}_{2} \in \mathrm{V}_{2}$ then $\mathrm{G}$ is called complete bipartite graph and it is denoted by $K_{\sigma 1, \sigma 2}$ where $\sigma 1 \& \sigma 2$ are respectively the restriction of $\sigma$ to $\mathrm{V}_{1}$ and $\mathrm{V}_{2}$

Definition 2.8. The complement of a fuzzy graph $\mathrm{G}(\sigma, \mu)$ is a subgraph $\bar{G}=$ $(\bar{\sigma}, \bar{\mu})$ where $\bar{\sigma}=\sigma$ and $\bar{\mu}(u, v)=\sigma(u) \wedge \sigma(v)-\mu(u, v)$ for all $u, v$ in $V$.

A fuzzy graph is self complementary if $G=\bar{G}$

Definition 2.9. The order $\mathrm{p}$ and size $\mathrm{q}$ of a fuzzy graph $\mathrm{G}(\sigma, \mu)$ is defined as $p=$ $\sum_{u \in V} \sigma(u)$ and $q=\sum_{(u, v) \in E} \mu(u, v)$.

Definition 2.10. The degree of the vertex $u$ is defined as the sum of weight of arc incident at $\mathrm{u}$, and is denoted by $\mathrm{d}(\mathrm{u})$.

Definition 2.11. A Path $\rho$ of a fuzzy graph $\mathrm{G}(\sigma, \mu)$ is a sequence of distinct nodes $\mathrm{v}_{1}, \mathrm{v}_{2}$, $\mathrm{v}_{3,}, \ldots \mathrm{v}_{\mathrm{n}}$ such that $\mu\left(v_{i-1}, v_{i}\right)>0$ where $1 \leq i \leq n$. A path is called a cycle if $u_{0}=$ $u_{n}$ and $n \geq 3$.

Definition 2.12. Let $\mathrm{u}, \mathrm{v}$ be two nodes in $\mathrm{G}(\sigma, \mu)$. If they are connected by means of a path $\rho$ then strength of that path is $\bigwedge_{i=i}^{n} \mu\left(u_{i-1}, v_{i}\right)$.

Definition 2.13. Two nodes that are joined by a path are said to be connected. The relation connected is reflexive, symmetric and transitive. If $\mathrm{u}$ and $\mathrm{v}$ are connected by means of length $\mathrm{k}$, then $\mu^{k}(u, v)=\sup \left\{\mu\left(u, v_{1}\right) \wedge \mu\left(v_{1}, v_{2}\right) \ldots \wedge \mu\left(v_{k-1}, v_{k}\right)\right.$ । $u, v_{1}, v_{2}, \ldots v$ in such path $\left.\rho\right\}$ 


\section{Case Study of Non Strong Arc in Cartesian Product of Fuzzy Graphs}

Definition 2.14. A Strongest path joining any two nodes $u, v$ is a path corresponding to maximum strength between $u$ and $v$. The strength of the strongest path is denoted by $\mu^{\infty}(u, v) . \mu^{\infty}(u, v)=\sup \left\{\mu^{k}(u, v) \mid \mathrm{k}=1,2,3 \ldots\right\}$

\section{Example 2.1.}

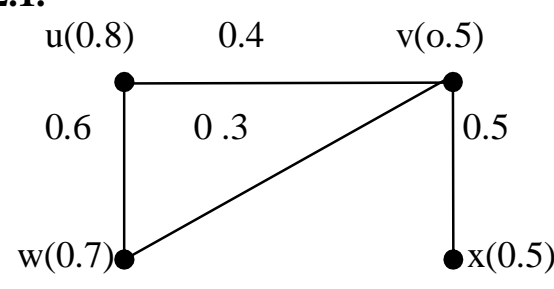

(a)

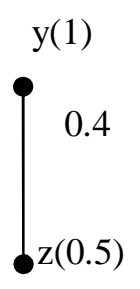

(b)

Figure 1:

In this fuzzy graph, Fig 1(a), $u=w, v, x$ is a w-x path of length 2 and strength is 0.3 . Another path of $\mathrm{w}-\mathrm{x}$ is $\mathrm{w}, \mathrm{u}, \mathrm{v}, \mathrm{x}$ of length 3 and strength is 0.4 .

But strength of the strongest path joining $\mathrm{w}$ and $\mathrm{x}$ is $\mu^{\infty}(w, x)=\sup \{0.3,0.4\}=0.4$

Definition 2.15. Let $\mathrm{G}(\sigma, \mu)$ be fuzzy graph. Let $\mathrm{x}$, y be two distinct nodes and $G^{\prime}$ be the fuzzy subgraph obtained by deleting the arc (x,y) that is $G^{\prime}\left(\sigma, \mu^{\prime}\right)$ where $\mu^{\prime}(x, y)=$ 0 and $\mu^{\prime}=\mu$ for all other. Then (x,y) is said to be fuzzy bridge in $\mathrm{G}$ if $\mu^{\prime \infty}(u, v)<$ $\mu^{\infty}(u, v)$ for some $u, v$ in $V$

Definition 2.16. A node is a fuzzy cut node of $\mathrm{G}(\sigma, \mu)$ if removal of it reduces the strength of the connectedness between some other pair of nodes. That is, $w$ is a fuzzy cut node of $\mathrm{G}(\sigma, \mu)$ iff there exist $\mathrm{u}, \mathrm{v}$ such that $\mathrm{w}$ is on every strongest path from $\mathrm{u}$ to $\mathrm{v}$.

Definition 2.17. An arc (u, v) of the fuzzy graph $\mathrm{G}(\sigma, \mu)$ is called an effective edge if $\mu(u, v)=\sigma(u) \wedge \sigma(v)$ and effective edge neighborhood of $\mathrm{u} \in V$ is $N_{e}(u)=\{v \in$ $V$ : edge $(u, v)$ is effective $\} . N_{e}[u]=N_{e}(u) \cup\{u\}$ is the closed neighbourhood of u. The minimum cardinality of effective neighborhood $\delta_{e}(G)=\min \left\{\left|N_{e}(u)\right| u \in V(G)\right\}$. Maximum cardinality of effective neighborhood $\Delta_{e}(G)=\max \left\{\left|N_{e}(u)\right| u \in V(G)\right\}$.

\section{Domination in fuzzy graphs using strong arcs}

Definition 2.18. An arc $(\mathrm{u}, \mathrm{v})$ of the fuzzy graph $\mathrm{G}(\sigma, \mu)$ is called a strong arc if $\mu(u, v)=\mu^{\infty}(u, v)$ else $\operatorname{arc}(\mathrm{u}, \mathrm{v})$ is called non strong. Strong neighborhood of $\mathrm{u} \in V$ is $N_{s}(u)=\{v \in V: \operatorname{arc}(u, v)$ is strong $\} . N_{s}[u]=N_{s}(u) \cup\{u\}$ is the closed neighborhood of $u$. The minimum cardinality of strong neighborhood $\delta_{s}(G)=\min \left\{\left|N_{s}(u)\right|: u \in\right.$ $V(G)\}$. Maximum cardinality of strong neighborhood $\Delta_{S}(G)=\max \left\{\left|N_{S}(u)\right|: u \in\right.$ $V(G)\}$.

Definition 2.19. Let $\mathrm{G}(\sigma, \mu)$ be a fuzzy graph. Let $\mathrm{u}$, $\mathrm{v}$ be two nodes of $\mathrm{G}(\sigma, \mu)$. We say that $\mathrm{u}$ dominates $\mathrm{v}$ if edge $(\mathrm{u}, \mathrm{v})$ is a strong arc. A subset $\mathrm{D}$ of $\mathrm{V}$ is called a dominating set of $\mathrm{G}(\sigma, \mu)$ if for every $v \in V-D$, there exists $u \in D$ such that u dominates $\mathrm{v}$. A dominating set $\mathrm{D}$ is called a minimal dominating set if no proper subset of $\mathrm{D}$ is a 


\section{C.Y. Ponnappan and V.Senthilkumar}

dominating set. The minimum fuzzy cardinality taken over all dominating sets of a graph $\mathrm{G}$ is called the strong arc domination number and is denoted by $\gamma_{s}(G)$ and the corresponding dominating set is called minimum strong arc dominating set.. The number of elements in the minimum strong arc dominating set is denoted by $\mathrm{n}\left[\gamma_{s}(G)\right]$

Example 2.20. In fig(i) $(\mathrm{a}),(\mathrm{u}, \mathrm{v}),(\mathrm{u}, \mathrm{w}),(\mathrm{v}, \mathrm{x})$ are strong $\operatorname{arcs}$ and $(\mathrm{v}, \mathrm{w})$ is non strong arc. $D_{1}=\{u, x\}, D_{2}=\{w, x\}, D_{3}=\{w, v\}, D_{4}=\{u, v\}$ are dominating sets. Also $D_{1}, D_{2}$, $\mathrm{D}_{3}, \mathrm{D}_{4}$ are minimal dominating sets. Therefore $\left|D_{1}\right|=0.8+0.5=1.3,\left|D_{2}\right|=0.7+$ $0.5=1.2,\left|D_{3}\right|=0.5+0.7=1.2$ and $\left|D_{4}\right|=0.8+0.5=1.3$. Therefore, $\min \{1.3,1.2,1.2,1.3\}=1.2$.

Hence $\mathrm{D}_{1}$ and $\mathrm{D}_{2}$ are minimum dominating sets. $\gamma_{s}=1.2$ and $\mathrm{n}\left[\gamma_{s}[G]=2\right.$.

\section{Case study of non strong arc in Cartesian product of fuzzy graph}

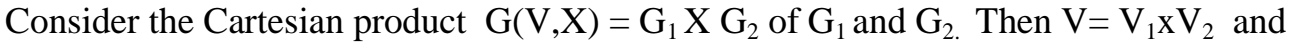
$\mathrm{X}=\left\{\left(\left(\mathrm{u}, \mathrm{u}_{2}\right),\left(\mathrm{u}, \mathrm{v}_{2}\right)\right) / \mathrm{u} \in \mathrm{V}_{1},\left(\mathrm{u}_{2}, \mathrm{v}_{2}\right) \in X_{2}\right\} \mathrm{U}\left\{\left(\left(\mathrm{u}_{1}, \mathrm{w}\right),\left(\mathrm{v}_{1}, \mathrm{w}\right)\right) / \mathrm{w} \in \mathrm{V}_{2},\left(\mathrm{u}_{2}, \mathrm{v}_{1}\right) \in \mathrm{X}_{1}\right\}$.

Definition 3.1. Let $\sigma_{i}$ be a fuzzy subset of $\mathrm{V}_{\mathrm{i}}$ and let $\mu_{i}$ be a fuzzy subset of $\mathrm{X}_{\mathrm{i}} \mathrm{i}=1,2$. Define the fuzzy subsets $\sigma_{1} X \sigma_{2}$ of $\mathrm{V}$ and $\mu_{1} X \mu_{2}$ of $\mathrm{X}$ as follows:

$\left(\sigma_{1} X \sigma_{2}\right)\left(u_{1}, u_{2}\right)=\min \left\{\sigma_{1}\left(u_{1}\right), \sigma_{2}\left(u_{2}\right)\right\} \forall\left(u_{1}, u_{2}\right) \in V$ $\left(\mu_{1} X \mu_{2}\right)\left(\left(u, u_{2}\right),\left(u, v_{2}\right)\right)=\min \left\{\sigma_{1}\left(u_{1}\right), \mu_{2}\left(u_{2}, v_{2}\right)\right\} \forall u \in V_{1} \forall\left(u_{2}, v_{2}\right) \in X_{2}$ and $\left(\mu_{1} X \mu_{2}\right)\left(\left(u_{1}, w\right),\left(v_{1}, w\right)\right)=\min \left\{\sigma_{2}(w), \mu_{1}\left(u_{1}, v_{1}\right)\right\} \forall w \in V_{2}$ and $\forall\left(u_{1}, v_{1}\right) \in X_{1}$ Then the fuzzy graph $\mathrm{G}\left(\sigma_{1} X \sigma_{2}, \mu_{1} X \mu_{2}\right)$ is said to be the Cartesian product of $G_{1}\left(\sigma_{1}, \mu_{1}\right)$ and $G_{2}\left(\sigma_{2}, \mu_{2}\right)$.

Theorem 3.2. If any two vertices of fuzzy graph $\mathrm{G}(\sigma, \mu)$ are connected by exactly one path then every arc of $\mathrm{G}(\sigma, \mu)$ are strong.

Proof: Let $\mathrm{G}(\sigma, \mu)$ be a connected fuzzy graph and let $\mathrm{n}$ be the number vertices of $\mathrm{G}$.

Take $\mathrm{n}=2$, there must be $\mathrm{u}$ and $\mathrm{v}$ adjoined by one arc (Since $\mathrm{G}$ is connected fuzzy graph ).Clearly, $\mu^{\infty}(u . v)=\sup \{\mu(u, v)\}=\mu(u, v)$. Therefore , arc (u, v) is strong.

Assume that $n>2$. In a fuzzy path, the $\mu^{\infty}(u . v)$ of any arc in the path will be same fuzzy value $\mu(u, v)$ of the arc $(u, v)$ since connected by one path. By the above argument ,evidently it is proved that $\mu^{\infty}(u . v)=\mu(u, v)$ for any number of arc in a given path .Hence all the arc are strong.

Theorems 3.3. Let $\mathrm{G}(\sigma, \mu)$ be a fuzzy cycle in which lowest fuzzy value of an arc occurs at more than once ,then that arc must be strong.

Proof: Let $\mathrm{G}(\sigma, \mu)$ be a fuzzy cycle as in figure (ii) and let $\mu(u, v) \leq \mu(v, w) \leq \mu(u, w)$

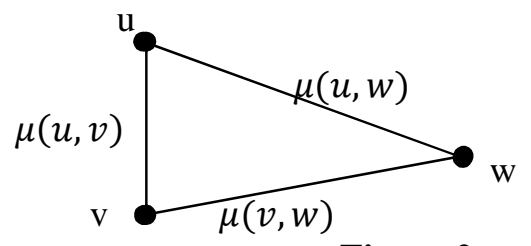

Figure 2:

Suppose , $\mu(u, v)<\mu(v, w) \leq \mu(u, w)$, then obviously arc (u, v) is non strong . 
Case Study of Non Strong Arc in Cartesian Product of Fuzzy Graphs

If not $\mu(u, v)=\mu(v, w)$, then $\mu^{\infty}(u \cdot v)=\sup \{\mu(u, v), \mu(v, w)\}=\mu(u, v)$

( Since there are two paths connecting $\mathrm{u}$ and $\mathrm{v}$ and $\mu(u, v)=\mu(v, w)$ )

Hence, arc (u, v) is strong ( by definition 3.1)

\section{Example 3.1.}

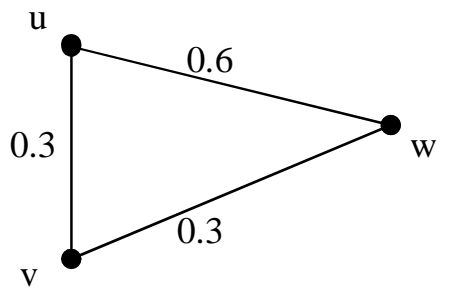

Figure 3:

$\mu(u, v)=\mu(v, w)=0,3$, then $\mu^{\infty}(u \cdot v)=\sup \{\mu(u, v), \mu(v, w)\}$ $\mu^{\infty}(u . v)=\sup \{0.3,0.3\}=0.3=\mu(u, v)$. Hence, $\operatorname{arc}(\mathrm{u}, \mathrm{v})$ is strong .

Note that, In the above example, all arc are strong.

Theorem 3.4. Let $\mathrm{G}\left(\sigma_{1} X \sigma_{2}, \mu_{1} X \mu_{2}\right)$ be the Cartesian product of fuzzy graph $G_{1}\left(\sigma_{1}, \mu_{1}\right)$ and $G_{2}\left(\sigma_{2}, \mu_{2}\right)$. If $G_{1}\left(\sigma_{1}, \mu_{1}\right)$ and $G_{2}\left(\sigma_{2}, \mu_{2}\right)$ doesn't have any non strong arc then $\mathrm{G}\left(\sigma_{1} X \sigma_{2}, \mu_{1} X \mu_{2}\right)$ doesn't have any non strong arc.

In other words, if all the arcs of $G_{1}$ and $G_{2}$ are strong then all the arcs of $G_{1} X$ are strong.

Proof: Assume that $G_{1}\left(\sigma_{1}, \mu_{1}\right)$ and $G_{2}\left(\sigma_{2}, \mu_{2}\right)$ are as in Fig. 4 (a), (b)

$$
\begin{array}{r}
\sigma_{1}\left(v_{1}\right) \\
\mu_{1}\left(u_{1}, v_{1}\right) \\
\sigma_{1}\left(u_{1}\right)
\end{array}
$$

(a)

$$
\begin{gathered}
\sigma_{2}\left(v_{2}\right) \\
\mu_{2}\left(u_{2}, v_{2}\right) \\
\sigma_{2}\left(u_{2}\right)
\end{gathered}
$$

(b)

Figure 4:

The Cartesian product of fuzzy graph $\mathrm{G}_{1} \times \mathrm{G}_{2}$ of $\mathrm{G}_{1}$ and $\mathrm{G}_{2}$ are drawn as in fig (iv)

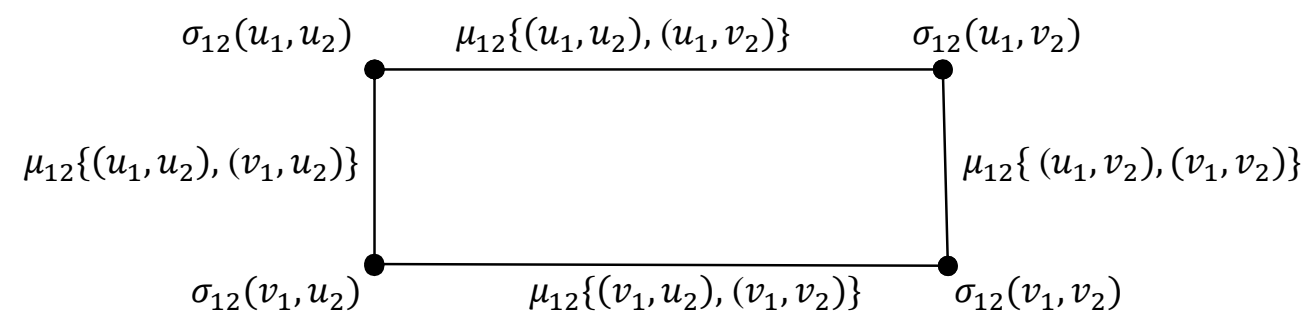

Figure 5: 


\section{C.Y. Ponnappan and V.Senthilkumar}

Here $G_{1}$ and $G_{2}$ are fuzzy path (or any fuzzy graph).

Clearly all the arcs of $\mathrm{G}_{1}$ and $\mathrm{G}_{2}$ are strong arc.(by theorem 3.1)

We have to prove that all the arcs of $\mathrm{G}_{1} \mathrm{X} \mathrm{\textrm {G } _ { 2 }}$ are strong. It is enough that we prove all the four arcs of fig (iv) are strong arc. The proof of the above hypothesis is discussed in the three four cases below.

\section{Case 1:}

Suppose $\sigma_{1}\left(u_{1}\right)<\mu_{2}\left(u_{2}, v_{2}\right)$ and $\sigma_{1}\left(v_{1}\right)<\mu_{2}\left(u_{2}, v_{2}\right)$

By definition 2.1, $\mu_{1}\left(u_{1}, v_{1}\right) \leq \sigma_{1}\left(v_{1}\right) \wedge \sigma_{1}\left(u_{1}\right)$ and $\mu_{2}\left(u_{2}, v_{2}\right) \leq \sigma_{2}\left(u_{2}\right) \wedge \sigma_{2}\left(v_{2}\right)$ Now, $\sigma_{1}\left(v_{1}\right)<\mu_{2}\left(u_{2}, v_{2}\right) \leq \sigma_{2}\left(u_{2}\right) \wedge \sigma_{2}\left(v_{2}\right) \quad($ by $(1)$

Hence, we must have $\sigma_{1}\left(u_{1}\right)<\sigma_{2}\left(u_{2}\right)$ and $\sigma_{1}\left(u_{1}\right)<\sigma_{2}\left(v_{2}\right)$

Similarly, $\sigma_{1}\left(v_{1}\right)<\sigma_{2}\left(u_{2}\right)$ and $\sigma_{1}\left(v_{1}\right)<\sigma_{s 2}\left(v_{2}\right)$

By definition of Cartesian product, a vertex $\mathrm{u}_{1}$ of $\sigma_{1}\left(u_{1}\right)$ of $\mathrm{G}_{1}$ can contribute two new

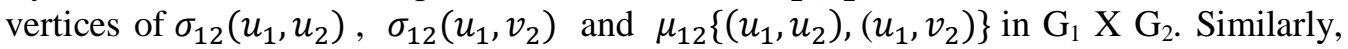
$\mathrm{v}_{1}$ can be done in the same method. As in fig (iv), following are the four arcs, whose fuzzy values are to be found.

Now, $\mu_{1} \mathrm{X} \mu_{2}\left\{\left(u_{1}, u_{2}\right),\left(u_{1}, v_{2}\right)\right\}=\min \left\{\sigma_{1}\left(u_{1}\right), \mu_{2}\left(u_{2}, v_{2}\right)\right\}=\sigma_{1}\left(u_{1}\right)$ (by (1))

$\mu_{1} \mathrm{X} \mu_{2}\left\{\left(v_{1}, u_{2}\right),\left(v_{1}, v_{2}\right)\right\}=\min \left\{\sigma_{1}\left(v_{1}\right), \mu_{2}\left(u_{2}, v_{2}\right)\right\}=\sigma_{1}\left(v_{1}\right)$ (by (1))

$\mu_{1} \mathrm{X} \mu_{2}\left\{\left(u_{1}, u_{2}\right),\left(v_{1}, u_{2}\right)\right\}=\min \left\{\mu_{1}\left(u_{1}, v_{1}\right), \sigma_{2}\left(u_{2}\right)\right\}=\mu_{1}\left(u_{1}, v_{1}\right)$

$\mu_{1} \mathrm{X} \mu_{2}\left\{\left(u_{1}, v_{2}\right),\left(v_{1}, v_{2}\right)\right\}=\min \left\{\mu_{1}\left(u_{1}, v_{1}\right), \sigma_{2}\left(v_{2}\right)\right\}=\mu_{1}\left(u_{1}, v_{1}\right)$

$\left\{\right.$ For, $\mu_{1}\left(u_{1}, v_{1}\right) \leq \sigma_{1}\left(u_{1}\right) \wedge \sigma_{1}\left(v_{1}\right)<\sigma_{2}\left(u_{2}\right) \wedge \sigma_{2}\left(v_{2}\right)$ by (2)

Hence, $\mu_{1}\left(u_{1}, v_{1}\right)<\sigma_{2}\left(u_{2}\right)$ and $\left.\mu_{1}\left(u_{1}, v_{1}\right)<\sigma_{2}\left(v_{2}\right)\right\}$

Now, the above arcs value are to be plotted in fig (iv). The fuzzy graph $G_{1} X G_{2}$ becomes

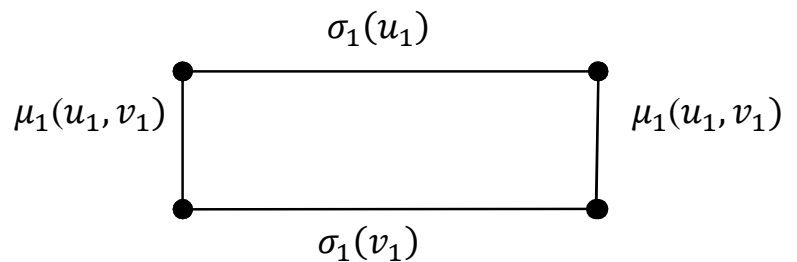

Figure 6:

Since, $\mu_{1}\left(u_{1}, v_{1}\right) \leq \sigma_{1}\left(u_{1}\right) \wedge \sigma_{1}\left(v_{1}\right)$ and (by theorem 3.2, Let $\mathrm{G}(\sigma, \mu)$ be a fuzzy cycle in which lowest fuzzy value of an arc occurs at more than once, then it must be strong arc.). Hence, all the arcs are strong in $\mathrm{G}_{1} \mathrm{X} \mathrm{G}_{2}$.

Case 2:

Suppose $\sigma_{1}\left(u_{1}\right)>\mu_{2}\left(u_{2}, v_{2}\right)$ and $\sigma_{1}\left(v_{1}\right)>\mu_{2}\left(u_{2}, v_{2}\right)$

$\mu_{1} \mathrm{X} \mu_{2}\left\{\left(u_{1}, u_{2}\right),\left(u_{1}, v_{2}\right)\right\}=\min \left\{\sigma_{1}\left(u_{1}\right), \mu_{2}\left(u_{2}, v_{2}\right)\right\}=\mu_{2}\left(u_{2}, v_{2}\right)$ ( by (3))

$\mu_{1} \mathrm{X} \mu_{2}\left\{\left(v_{1}, u_{2}\right),\left(v_{1}, v_{2}\right)\right\}=\min \left\{\sigma_{1}\left(v_{1}\right), \mu_{2}\left(u_{2}, v_{2}\right)\right\}=\mu_{2}\left(u_{2}, v_{2}\right)$ (by (3))

$\left.\mu_{1} \mathrm{X} \mu_{2}\left\{\left(u_{1}, u_{2}\right),\left(v_{1}, u_{2}\right)\right\}=\min \left\{\mu_{1}\left(u_{1}, v_{1}\right), \sigma_{2}\left(u_{2}\right)\right\}\right\}$

$\left.\mu_{1} \mathrm{X} \mu_{2}\left\{\left(u_{1}, v_{2}\right),\left(v_{1}, v_{2}\right)\right\}=\min \left\{\mu_{1}\left(u_{1}, v_{1}\right), \sigma_{2}\left(v_{2}\right)\right\}\right\}=$ any fuzzy value but $\geq$

$\mu_{2}\left(u_{2}, v_{2}\right)$ (or $) \geq \mu_{1}\left(u_{1}, v_{1}\right)$.

For,

If $\mu_{1}\left(u_{1}, v_{1}\right) \geq \mu_{2}\left(u_{2}, v_{2}\right) \quad$ then we must have $\sigma_{2}\left(u_{2}\right) \geq \mu_{2}\left(u_{2}, v_{2}\right), \sigma_{2}\left(v_{2}\right) \geq$ $\mu_{2}\left(u_{2}, v_{2}\right)$ and if $\mu_{1}\left(u_{1}, v_{1}\right) \leq \mu_{2}\left(u_{2}, v_{2}\right)$ then we must have $\sigma_{2}\left(u_{2}\right) \geq \mu_{1}\left(u_{1}, v_{1}\right)$, $\sigma_{2}\left(v_{2}\right) \geq \mu_{1}\left(u_{1}, v_{1}\right)$ 
Case Study of Non Strong Arc in Cartesian Product of Fuzzy Graphs

In this case $\mu_{2}\left(u_{2}, v_{2}\right)$ or $\mu_{1}\left(u_{1}, v_{1}\right)$ is the lowest fuzzy value of other arcs of $\mathrm{G}_{1} \mathrm{X} \mathrm{G}_{2}$ and it can occur more than once. By theorem 3.2, all the arcs of $\mathrm{G}_{1} \mathrm{X} \mathrm{G}_{2}$ are strong.

\section{Case 3:}

Suppose, $\sigma_{1}\left(u_{1}\right)<\mu_{2}\left(u_{2}, v_{2}\right)$ and $\sigma_{1}\left(v_{1}\right)>\mu_{2}\left(u_{2}, v_{2}\right)$

$\mu_{1} \mathrm{X} \mu_{2}\left\{\left(u_{1}, u_{2}\right),\left(u_{1}, v_{2}\right)\right\}=\min \left\{\sigma_{1}\left(u_{1}\right), \mu_{2}\left(u_{2}, v_{2}\right)\right\}=\sigma_{1}\left(u_{1}\right)$ (by (4) )

$\mu_{1} \mathrm{X} \mu_{2}\left\{\left(v_{1}, u_{2}\right),\left(v_{1}, v_{2}\right)\right\}=\min \left\{\sigma_{1}\left(v_{1}\right), \mu_{2}\left(u_{2}, v_{2}\right)\right\}=\mu_{2}\left(u_{2}, v_{2}\right)$ (by (4) )

$\mu_{1} \mathrm{X} \mu_{2}\left\{\left(u_{1}, u_{2}\right),\left(v_{1}, u_{2}\right)\right\}=\min \left\{\mu_{1}\left(u_{1}, v_{1}\right), \sigma_{2}\left(u_{2}\right)\right\}=\mu_{1}\left(u_{1}, v_{1}\right)$

$\mu_{1} \mathrm{X} \mu_{2}\left\{\left(u_{1}, v_{2}\right),\left(v_{1}, v_{2}\right)\right\}=\min \left\{\mu_{1}\left(u_{1}, v_{1}\right), \sigma_{2}\left(v_{2}\right)\right\}=\mu_{1}\left(u_{1}, v_{1}\right)$

For,

Now, $\mu_{1}\left(u_{1}, v_{1}\right) \leq \sigma_{1}\left(u_{1}\right)<\mu_{2}\left(u_{2}, v_{2}\right)$.

Therefore, we must have $\mu_{1}\left(u_{1}, v_{1}\right)<\sigma_{2}\left(u_{2}\right)$ and $\mu_{1}\left(u_{1}, v_{1}\right)<\sigma_{2}\left(v_{2}\right)$. From (4), we also have $\sigma_{2}\left(u_{2}\right)>\sigma_{1}\left(u_{1}\right)$, and $\sigma_{2}\left(v_{2}\right)>\sigma_{1}\left(u_{1}\right)$.

In this case, $\mu_{1}\left(u_{1}, v_{1}\right)$ is the lowest value of all other arcs of $\mathrm{G}_{1} \mathrm{X} \mathrm{G}_{2}$ and this arc occurs more than once in fuzzy cycle. Hence all the arcs are strong.

\section{Case 4:}

Suppose $\sigma_{1}\left(u_{1}\right)=\mu_{2}\left(u_{2}, v_{2}\right)$ and $\sigma_{1}\left(v_{1}\right)<\mu_{2}\left(u_{2}, v_{2}\right)$,

$\sigma_{1}\left(u_{1}\right)<\mu_{2}\left(u_{2}, v_{2}\right)$ and $\sigma_{1}\left(v_{1}\right)=\mu_{2}\left(u_{2}, v_{2}\right)$ and

$\sigma_{1}\left(u_{1}\right)=\mu_{2}\left(u_{2}, v_{2}\right)$ and $\sigma_{1}\left(v_{1}\right)=\mu_{2}\left(u_{2}, v_{2}\right)$.

Evidently, this case can be proved by the above three cases.

Hence, if all the arcs of $G_{1}$ and $G_{2}$ are strong then all the arcs of $G_{1} X G_{2}$ are strong.

Example for case (i)

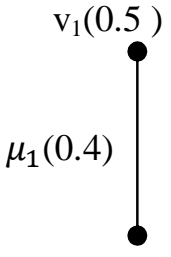

$u_{1}(0.6)$

$\mathrm{G}_{1}$

Example for Case (ii)

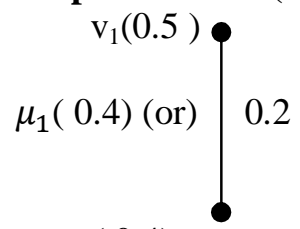

$\mathrm{u}_{1}(0.4)$

$\mathrm{G}_{1}$

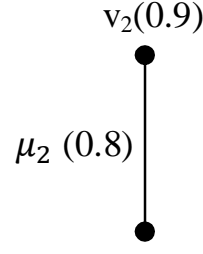

$\mathrm{u}_{2}(1)$

$\mathrm{G}_{2}$

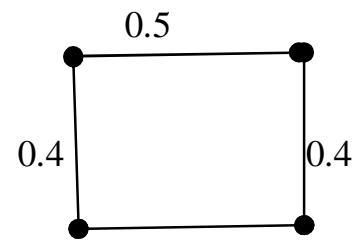

0.6

$\mathrm{G}_{1} \mathrm{XG}_{2}$

Figure 7:

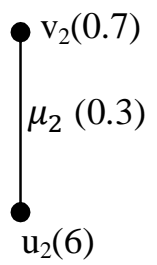

$\mathrm{G}_{2}$

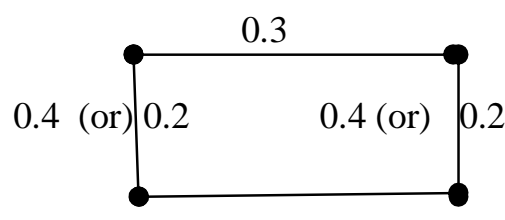

0.3

$\mathrm{G}_{1} \mathrm{XG}_{2}$

Figure 8: 
Example for Case (iii)

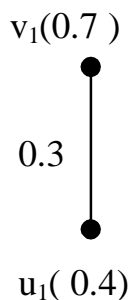

$\mathrm{G}_{1}$

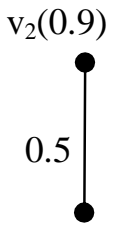

$\mathrm{u}_{2}(0.6)$

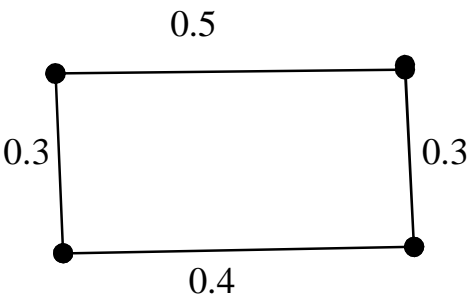

$\mathrm{G}_{1} \times \mathrm{G}_{2}$

Figure 9:

Examples of the above three cases shows that if all the arcs of $\mathrm{G}_{1}$ and $\mathrm{G}_{2}$ are strong then all the arcs of $\mathrm{G}_{1} \mathrm{X} \mathrm{G}_{2}$ are strong.

Note 3.5. Converse need not be true.

If all the arcs in $G_{1} X G_{2}$ are strong, it is not necessary that all arcs in $G_{1}$ or in $G_{2}$ must be strong. In other words, non strong arc can exist in $G_{1}$ or in $G_{2}$.

Note 3.6. If either $G_{1}$ or $G_{2}$ have an non strong arc then $G_{1} X G_{2}$ need not have non strong arc.

But, if both have non strong arc then $\mathrm{G}_{1} \mathrm{X} \mathrm{G}_{2}$ must have non strong arc.

The following theorem proved that existence of non strong in $G_{1} X G_{2}$.

Theorem 3.7. Let $G_{1}$ and $G_{2}$ be two fuzzy graph of which at least one has non strong arc, then the existing non strong arc in $\mathrm{G}_{1} \mathrm{X} \mathrm{G}_{2}$ depends on the fuzzy value of the vertices of $G_{1}$ or $G_{2}$ depends upon the non strong arc that appears in $G_{2}$ or in $G_{1}$ respectively.

That is, though $G_{1}$ or $G 2$ have non strong arc, it is not necessary that $G_{1} X_{2}$ must have non strong arc.

Proof: To prove this hypothesis, we can take the minimum consideration of fuzzy graph as follows.

Let $G_{1}\left(\sigma_{1}, \mu_{1}\right)$ be fuzzy graph which has one non strong and let $G_{2}\left(\sigma_{2}, \mu_{2}\right)$ be another fuzzy graph which has no non strong respectively in fig (v) (a) and (b)

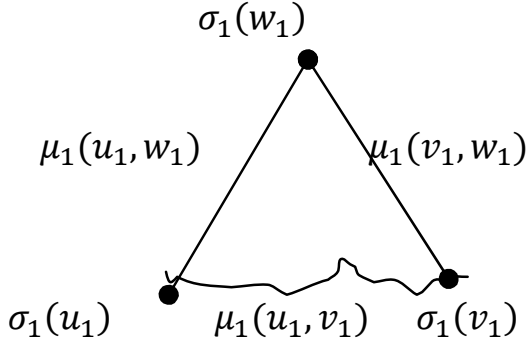

(a) $\mathrm{G}_{1}$

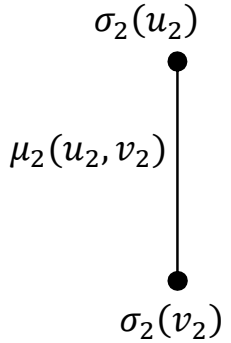

(b) $\mathrm{G}_{2}$

Figure 10: 


\section{Case Study of Non Strong Arc in Cartesian Product of Fuzzy Graphs}

Assume that $\mu_{1}\left(u_{1}, v_{1}\right)<\mu_{1}\left(v_{1}, w_{1}\right) \leq \mu_{1}\left(u_{1}, w_{1}\right)$. Therefore, $\mu_{1}\left(u_{1}, v_{1}\right)$ is a non strong arc $\mathrm{G}_{1}$ ( by theorem 3.1), there is no non strong in $\mathrm{G}_{2}$

Claim: The existing non strong $\mu_{1}\left(u_{1}, v_{1}\right)$ arc in $\mathrm{G}_{1} \mathrm{X} \mathrm{G}_{2}$ depends on $\sigma_{2}\left(u_{2}\right)$ and $\sigma_{2}\left(v_{2}\right)$ The proof of this claim to be discussed in three cases.

Case 1: Let $\mu_{1}\left(u_{1}, v_{1}\right)<\sigma_{2}\left(u_{2}\right)$ and $\mu_{1}\left(u_{1}, v_{1}\right)<\sigma_{2}\left(v_{2}\right)$

Let $\mathrm{G}\left(\sigma_{1} X \sigma_{2}, \mu_{1} X \mu_{2}\right)$ be the Cartesian product of fuzzy graph $G_{1}\left(\sigma_{1}, \mu_{1}\right)$ and $G_{2}\left(\sigma_{2}, \mu_{2}\right)$ are drawn in Fig 11 .

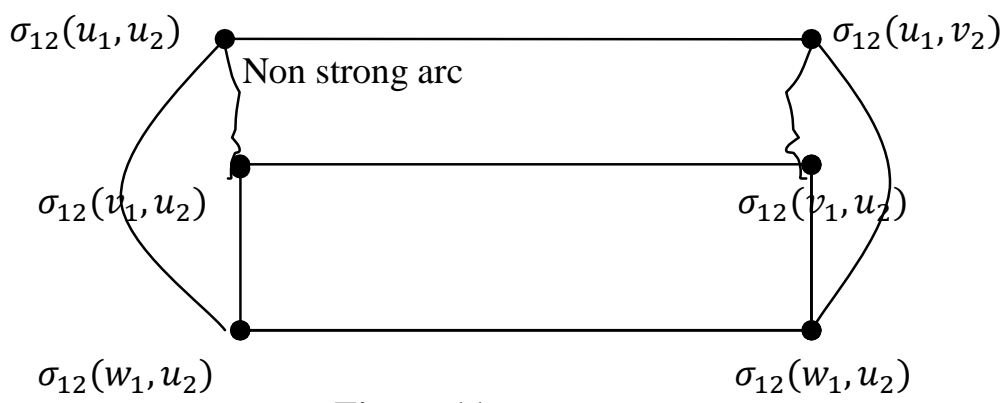

Figure 11:

Now, fuzzy value of the arc of $\mathrm{G}_{1} \mathrm{X} \mathrm{G}_{2}$ are found as follows.

$\mu_{1} \mathrm{X} \mu_{2}\left\{\left(u_{1}, u_{2}\right),\left(v_{1}, u_{2}\right)\right\}=\min \left\{\sigma_{2}\left(u_{2}\right), \mu_{1}\left(u_{1}, v_{1}\right)\right\}=\mu_{1}\left(u_{1}, v_{1}\right) \quad$ (by (i))

$\mu_{1} \mathrm{X} \mu_{2}\left\{\left(v_{1}, u_{2}\right),\left(w_{1}, u_{2}\right)\right\}=\min \left\{\sigma_{2}\left(u_{2}\right), \mu_{1}\left(v_{1}, w_{1}\right)\right\}=\mu_{1}\left(v_{1}, w_{1}\right)$ (or) $\sigma_{2}\left(u_{2}\right)$

(Since, $\mu_{1}\left(u_{1}, v_{1}\right)<\mu_{1}\left(v_{1}, w_{1}\right) \leq \mu_{1}\left(u_{1}, w_{1}\right)$ and by(i))

$\mu_{1} \mathrm{X} \mu_{2}\left\{\left(u_{1}, u_{2}\right),\left(w_{1}, u_{2}\right)\right\}=\min \left\{\mu_{1}\left(u_{1}, w_{1}\right), \sigma_{2}\left(u_{2}\right)\right\}=\mu_{1}\left(u_{1}, w_{1}\right)$ (or) $\sigma_{2}\left(u_{2}\right)$.

These three fuzzy arc formed in one of the fuzzy cycle in $\mathrm{G}_{1} \mathrm{X}_{2}$ as in fig (v), in which $\mu_{1}\left(u_{1}, v_{1}\right)$ is the lowest value and this becomes non strong, since $\mu_{1}\left(u_{1}, v_{1}\right)<$ $\sigma_{2}\left(u_{2}\right)$.

Hence, there is one non strong exist in $\mathrm{G}_{1} \mathrm{X} \mathrm{G}_{2}$ when non strong $\mu_{1}\left(u_{1}, v_{1}\right)$ arc in $\mathrm{G}_{1}$ depends on $\sigma_{2}\left(u_{2}\right)$ such that $\mu_{1}\left(u_{1}, v_{1}\right)<\sigma_{2}\left(u_{2}\right)$.

Similarly, $\mu_{1} \mathrm{X} \mu_{2}\left\{\left(u_{1}, v_{2}\right),\left(v_{1}, v_{2}\right)\right\}=\min \left\{\sigma_{2}\left(v_{2}\right), \mu_{1}\left(u_{1}, v_{1}\right)\right\}=\mu_{1}\left(u_{1}, v_{1}\right)$ (by (i) ) $\mu_{1} \mathrm{X} \mu_{2}\left\{\left(v_{1}, v_{2}\right),\left(w_{1}, v_{2}\right)\right\}=\min \left\{\sigma_{2}\left(v_{2}\right), \mu_{1}\left(v_{1}, w_{1}\right)\right\}=\mu_{1}\left(v_{1}, w_{1}\right)$ (or) $\sigma_{2}\left(v_{2}\right)$

(Since, $\mu_{1}\left(u_{1}, v_{1}\right)<\mu_{1}\left(v_{1}, w_{1}\right) \leq \mu_{1}\left(u_{1}, w_{1}\right)$ and by(i))

$\mu_{1} \mathrm{X} \mu_{2}\left\{\left(u_{1}, v_{2}\right),\left(w_{1}, v_{2}\right)\right\}=\min \left\{\mu_{1}\left(u_{1}, w_{1}\right), \sigma_{2}\left(v_{2}\right)\right\}=\mu_{1}\left(u_{1}, w_{1}\right)$ (or) $\sigma_{2}\left(v_{2}\right)$

These three fuzzy arc forms another fuzzy cycle in $\mathrm{G}_{1} \mathrm{X} \mathrm{G}_{2}$ as in fig (vi), in which $\mu_{1}\left(u_{1}, v_{1}\right)$ is the lowest value and becomes non strong arc, since $\mu_{1}\left(u_{1}, v_{1}\right)<\sigma_{2}\left(v_{2}\right)$.

From the above argument, it is concluded that the non strong arc existing in $\mathrm{G}_{1} \mathrm{X}$ $\mathrm{G}_{2}$ depends on $\sigma_{2}\left(u_{2}\right)$ and $\sigma_{2}\left(v_{2}\right)$ of $\mathrm{G}_{2}$ where non strong $\mu_{1}\left(u_{1}, v_{1}\right)$ arc is in $\mathrm{G}_{1}$ and therefore there are two non strong arcs in $\mathrm{G}_{1} \mathrm{X} \mathrm{G}_{2}$, since non strong $\operatorname{arc} \mu_{1}\left(u_{1}, v_{1}\right)$ < $\sigma_{2}\left(u_{2}\right)$ and $\mu_{1}\left(u_{1}, v_{1}\right)<\sigma_{2}\left(v_{2}\right)$.

Case 2: Let $\mu_{1}\left(u_{1}, v_{1}\right)>\sigma_{2}\left(u_{2}\right)$ and $\mu_{1}\left(u_{1}, v_{1}\right)>\sigma_{2}\left(v_{2}\right)$

Now, fuzzy value of the arc of $\mathrm{G}_{1} \times \mathrm{G}_{2}$ are found as follws.

$\mu_{1} \mathrm{X} \mu_{2}\left\{\left(u_{1}, u_{2}\right),\left(v_{1}, u_{2}\right)\right\}=\min \left\{\sigma_{2}\left(u_{2}\right), \mu_{1}\left(u_{1}, v_{1}\right)\right\}=\sigma_{2}\left(u_{2}\right)$ (by (ii)) 


\section{C.Y. Ponnappan and V.Senthilkumar}

$\mu_{1} \mathrm{X} \mu_{2}\left\{\left(v_{1}, u_{2}\right),\left(w_{1}, u_{2}\right)\right\}=\min \left\{\sigma_{2}\left(u_{2}\right), \mu_{1}\left(v_{1}, w_{1}\right)\right\}=\sigma_{2}\left(u_{2}\right)$

(Since, $\mu_{1}\left(u_{1}, v_{1}\right)<\mu_{1}\left(v_{1}, w_{1}\right) \leq \mu_{1}\left(u_{1}, w_{1}\right)$ and by(i) )

$\mu_{1} X \mu_{2}\left\{\left(u_{1}, u_{2}\right),\left(w_{1}, u_{2}\right)\right\}=\min \left\{\mu_{1}\left(u_{1}, w_{1}\right), \sigma_{2}\left(u_{2}\right)\right\}=\mu_{1}\left(u_{1}, w_{1}\right)$.

These three fuzzy arc forms fuzzy cycle in $\mathrm{G}_{1} \mathrm{XG}_{2}$ as in fig (vi), in which all the arc value becomes strong since $\mu_{1}\left(u_{1}, v_{1}\right)>\sigma_{2}\left(u_{2}\right)$ and $\sigma_{2}\left(u_{2}\right)$ is the lowest value that occurs more than once in $\mathrm{G}_{1} \times \mathrm{G}_{2}$. Similarly, the same result provided by non strong arc $\mu_{1}\left(u_{1}, v_{1}\right)$ in $\mathrm{G}_{1}$ by the Cartesian product with $\sigma_{2}\left(v_{2}\right)$.

Therefore, in this case no non strong arc exist in $\mathrm{G}_{1} \mathrm{X} \mathrm{G}_{2}$, since $\mu_{1}\left(u_{1}, v_{1}\right)>$ $\sigma_{2}\left(u_{2}\right)$ and $\mu_{1}\left(u_{1}, v_{1}\right)>\sigma_{2}\left(v_{2}\right)$

Case 3: Let $\mu_{1}\left(u_{1}, v_{1}\right)=\sigma_{2}\left(u_{2}\right)$ and $\mu_{1}\left(u_{1}, v_{1}\right)=\sigma_{2}\left(v_{2}\right)$

Now, fuzzy value of the arc of $\mathrm{G}_{1} \times \mathrm{G}_{2}$ are found as follows.

$\mu_{1} \mathrm{X} \mu_{2}\left\{\left(u_{1}, u_{2}\right),\left(v_{1}, u_{2}\right)\right\}=\min \left\{\sigma_{2}\left(u_{2}\right), \mu_{1}\left(u_{1}, v_{1}\right)\right\}=\sigma_{2}\left(u_{2}\right)=\mu_{1}\left(u_{1}, v_{1}\right)$ (by (ii))

$\mu_{1} \mathrm{X} \mu_{2}\left\{\left(v_{1}, u_{2}\right),\left(w_{1}, u_{2}\right)\right\}=\min \left\{\sigma_{2}\left(u_{2}\right), \mu_{1}\left(v_{1}, w_{1}\right)\right\}=\sigma_{2}\left(u_{2}\right)=\mu_{1}\left(u_{1}, v_{1}\right)$

(Since, $\mu_{1}\left(u_{1}, v_{1}\right)<\mu_{1}\left(v_{1}, w_{1}\right) \leq \mu_{1}\left(u_{1}, w_{1}\right)$ and by(ii))

$\mu_{1} X \mu_{2}\left\{\left(u_{1}, u_{2}\right),\left(w_{1}, u_{2}\right)\right\}=\min \left\{\mu_{1}\left(u_{1}, w_{1}\right), \sigma_{2}\left(u_{2}\right)\right\}=\sigma_{2}\left(u_{2}\right)=\mu_{1}\left(u_{1}, v_{1}\right)$.

These three arc with same fuzzy value in fuzzy cycle in $\mathrm{G}_{1} \mathrm{X} \mathrm{G}_{2}$ are strong

(by theorem 3.2), similarly the same result exist for other three arcs.

Therefore, in this case also no non strong arc exist in $\mathrm{G}_{1} \mathrm{X} \mathrm{G}_{2}$,

Case 4: Let $\mu_{1}\left(u_{1}, v_{1}\right)<\sigma_{2}\left(u_{2}\right)$ and $\mu_{1}\left(u_{1}, v_{1}\right) \geq \sigma_{2}\left(v_{2}\right)$

From the above cases, in case (1),there is one non strong arc existing in $\mathrm{G}_{1} \mathrm{X} \mathrm{G}_{2}$ when $\mu_{1}\left(u_{1}, v_{1}\right)<\sigma_{2}\left(u_{2}\right)$ and in case (2), case (3) and case (4), all the $\operatorname{arcs}$ of $\mathrm{G}_{1} \mathrm{X} \mathrm{G}_{2}$ are strong, when $\mu_{1}\left(u_{1}, v_{1}\right) \geq \sigma_{2}\left(v_{2}\right)$.

From the above four cases, it is concluded that non strong arc exist in $\mathrm{G}_{1} \mathrm{X} \mathrm{G}_{2}$ only when fuzzy value of non strong arc of $\mathrm{G}_{1}\left(\right.$ or $\left.\mathrm{G}_{2}\right)$ must be greater than the degree value of the vertex of $\mathrm{G}_{2}$ ( or $\mathrm{G}_{1}$ ) respectively. Hence, the existing non strong arc in $\mathrm{G}_{1}$ $X G_{2}$ depends on the fuzzy value of the vertices of $G_{1}$ or $G_{2}$ depending upon the non strong arc that appears in $G_{2}$ or in $G_{1}$ respectively.

Corollary 3.8. The number of non strong arc of $G_{1} X G_{2}=m_{1} P_{2}+m_{2} P_{1}$ where,

$\mathbf{m}_{1}$ - number of vertex of $G_{1}$, whose fuzzy value must greater than fuzzy value of non strong arc in other fuzzy graph $\mathrm{G}_{2}$

$\mathbf{m}_{2}$ - number of vertex of $G_{2}$, whose fuzzy value must greater than fuzzy value of non strong arc in other fuzzy graph $\mathrm{G}_{1}$

$\mathbf{P}_{\mathbf{1}}$ - number of non strong arc in $\mathrm{G}_{1}$

$\mathbf{P}_{2}$ - number of non strong arc in $\mathrm{G}_{2}$

\section{Conclusion}

In this paper, we discussed the condition for non strong arc to appear in Cartesian product of fuzzy graph, fuzzy path and fuzzy cycle etc. and deduced the formula for finding non strong arc in Cartesian product of fuzzy graph. Next our aim is to find the domination number of Cartesian product fuzzy graph and various type fuzzy graph by using strong arc.

Acknowledgement. Authors would like to thank to the referees for their helpful comments for improvement of the quality of the paper. 


\section{Case Study of Non Strong Arc in Cartesian Product of Fuzzy Graphs}

\section{REFERENCES}

1. K.R.Bhutani and A.Rosenfeld, Strong arcs in fuzzy graph, Information Sciences, 152 (2003) 319-322.

2. K.R.Bhutani, J.Mordeson and A.Rosenfeld, On degrees of end node and cut node in fuzzy graphs, Iranian Journal of Fuzzy systems, 1(1) (2004) 57-64.

3. F.Harary, Graph Theory, Addison Wesley, Reading, MA (1969).

4. J.N.Monderson and P.Nair, Fuzzy graphs and fuzzy hypergraphs, Physica-Verlag.

5. M.Pal, Intersection graphs: An introduction, Annals of Pure and Applied Mathematics, 4(1) (2013) 43-91.

6. M.Pal and H.Rashmanlou, Irregular interval -valued fuzzy graph, Annals of Pure and Applied Mathematics, 3(1) (2013) 56-66.

7. S.Methew and M.S.Sunitha, Types of arcs in fuzzy graph, Information Sciences, 179 (2009) 1760-1768.

8. A.Nagoorgani and V.T.Chandrasekaran, First Look at Fuzzy Graph Theory.

9. A.Nagoorgani and V.T.Chandrasekaran, Domination in fuzzy graph, Adv. In Fuzzy Set \& Systems, 1(1) (2006) 17-26.

10. O.Ore, Theory of Graphs. Amer. Math. Soc. Colloq. Publ. 38, (1962).

11. A.Rosenfeld, Fuzzy graphs. In: Zadeh, L.A., Fu, K.S., Shimura, M. Eds.., Fuzzy Sets and Their Applications. Academic Press, New York, (1975).

12. A.Somasundaram and S.Somasundaram, Domination in fuzzy graph-1, Pattern Recognition Letter, 19 (9) (1998) 787-791.

13. C.Y.Ponnappan, P.Surilinathan and S.Basheer Ahamed, Edge domination in fuzzy graph, new approach, International Journal of IT, Engg. Applied Sciences Research, 4(1) (2015).

14. S.Samanta and M.Pal, Irregular bipolar fuzzy graphs, International journal of Appllications of Fuzzy Sets and Artificial Intelligence, 2 (2012) 91-102.

15. S.Samanta, M.Pal and A.Pal, New concepts of fuzzy planar graph, International Journal of Advanced Research in Artificial Intelligence, 3(1) (2014) 52-59.

16. S.Samanta and M.Pal, Fuzzy planar graphs, IEEE Transaction on Fuzzy System, 23(6) (2015) $1936-1942$.

17. S.Samanta and M.Pal, Fuzzy $k$-competition graphs and $p$-competition fuzzy graphs, Fuzzy Inf. Eng., 5(2) (2013) 191-204.

18. H.Rashmanlou and M.Pal, Antipodal interval-valued fuzzy graphs, International Journal of Applications of Fuzzy Sets and Artificial Intelligence, 3 (2013) 107-130.

19. S.Sahoo and M.Pal, Intuitionistic fuzzy tolerance graphs with application, J. Appl. Math. Comput., 55 (2017) 495-511.

20. S.Samanta, M.Pal, R.A.Borzooei and H.Rashmanlou, Vague graphs and strengths, Journal of Intelligent and Fuzzy Systems, 30 (2016) 3675-3680.

21. L.A.Zadeh, Fuzzy set, Information and Control, 8 (1965) 338-353. 